\title{
ALGORITHM AND SOFTWARE FOR OPERATIVE CALCULATIONS OF THE SHORT CIRCUITS MODES
}

\author{
Stanislav V. Borisov ${ }^{1}$, Sergey N. Kladiev ${ }^{1, a}$, Konstantin V. Obraztsov ${ }^{1}$ \\ ${ }^{1}$ National Research Tomsk Polytechnic University, 634050 Tomsk, Russia
}

\begin{abstract}
Smart power grids provide efficient control of power flows or other grid variables, such as voltages or short circuit currents. Most essential the control has become, if the consumers are electrical systems of high power capacity. These systems are able to reconfigure the grid structure for emergency situations. The developed algorithm is intended for carrying out operational calculations of short-circuits modes in electrical grids of voltage $0,4 \mathrm{kV}$ to $220 \mathrm{kV}$. In the calculation the following methods have been used: methods of node voltages, method of superposition and method of branch expansion to form the node voltage matrix according to the graphs theory. In the software the short circuit inflow from all electrical motors and nodes with generalized load is taken into account as opposed to other algorithms. The calculated circuit can have up to 700 nodes; there is ability to decrease number of branches and nodes and to form the calculative model of the grid part. Calculation algorithm of the short circuit currents is implemented as a sequence of software calculations. Software of systems and grids, in which short circuits are altered, allows carrying out operational calculations of the short circuit modes to adjust settings of relays protection devices, to check electrical equipment and to replace it, to choose optimal operational circuits. Actuality of the calculations is caused by need of the software implementation to maintain, upgrade and design the electrical grids of enterprises that have their own power plants.
\end{abstract}

\section{Introduction}

Many power supply systems are in development, in these the short circuits (SC) currents are changing quite often [1]. The SC modes calculation are needed for correction and adjusting the settings of the relays protection and automation devices; for checking the electric equipment that is installed or replaced; for choosing optimal operational circuits, which are often chosen without appropriate substantiation [3]. Within the automated control system of the technological procedures (ACS TP) a program for calculation of the SC currents is needed, which is suitable for carrying out the operational calculations. This program should have the corresponding data base, in order to whose check a special program is developed.

The developed software for calculation of SC modes allows to calculate the values of SC currents in required nodes of electric grids, residual voltages in all grid's nodes and the current distribution in the branches of equivalent circuit. In the software of the short circuit calculation the current inflow from all electrical motors of the enterprise is taken into account as opposed to other programs.

The need of the transients calculations in power supply systems is caused by the fact that their behaviour has influence on operational reliability of these systems and their components. The transients are divided into normal

a Corresponding author : kladiev@tpu.ru 


\section{MATEC Web of Conferences}

and abnormal (emergency). As normal can be considered the transients caused by impact load, which cause the voltage fluctuations, as well as by starting electric motors, which influence the operation stability of other already functioning electric motors, which can lead to an emergency and stop of manufacturing. As abnormal transients can be considered operation modes of the short circuit and self-starting of electric motors after power supply failures and power-downs of various duration.

By controlling the transients the emergency stop of manufacturing procedure caused by non-starting of electric motors can be prevented. To realize it a protection selectivity map is composed taking into account automatic starting of electric motors. The settings of the maximum current and minimum voltage protections and starting time in automatic mode have to be calculated taking into account the transients. It prevents false triggering of protections in control stations, which cause disabling the automatic starting.

The transients behavior in power supply systems is influenced as well by: grid configuration; operative connection circuit of the whole district; characteristics of the electric motors and the machinery driven by them; remoteness of the load nodes from power supply (PS); automatic equipment, which controls the induction motors (IM) and synchronous motors (SM), substations and in grids of voltage $0.4 \div 35 \mathrm{kV}$. Therefore «improving» of the transients' behavior, i.e. reducing their influence to operation modes of the power supply system can be carried out by means of:

- changing the circuit of power supply, which was chosen with no account of the transients behavior (in design, expansion and modernization phases of life-cycle);

- choosing optimal operative circuit, settings of the automatic and relay protection devices; improvement of these devices in any time.

And to make the planned actions and recommendations more reliable the accurate and valid computing methods of the transients should be used [6].

For further development of the ACS TP for its mathematical support (MS) a data base needs to be implemented, in which technical and operation parameters of all electric motors, driven machinery and the relay protection and automatic control devices in normal and emergency modes. Another component of MS is software for calculation of steady-state and transient modes to realize operative and perspective calculations. One of components of this MS is the proposed software.

\section{Problem statement}

The software should be able to take into account the short current inflow from all electrical motors of the enterprise, the operative changes in circuit of the power supply and to calculate the residual voltages in nodes and the currents in branches of the circuit.

Problem definition. Before calculating the SC modes a data base should be generated: an «array» of branch parameters is proposed to be generated, which contains initial data of power lines, transformers, system, electric motors, generators, reactors and generalized load nodes (an example of such «array» is shown in table 1).

In table 1 there are following symbols:

EPSS is Electric Power Supply System;

$O H P L$ is Overhead Power Line;

$T$ is Power Transformer;

$B S B$ is Bus Section Breaker;

$P C L$ is Power Cable Line;

$G L N$ is Generalized Load Node.

By the software should be calculated: the total short current for a given node, residual voltages in all nodes of the system, currents in all components of the circuit. Intermediate calculations can be fulfilled in absolute or relative units.

The used algorithm needs to form a matrix of node impedances (or matrix of short currents) and to calculate two modes: a normal and a purely «emergency» (additional), which are superimposed on each other. To solve the task a model is used that is based on a system of linear equations in matrix form. These equations can be considered as linear only for specific time instant of the transient. 
Table 1. System parameters

\begin{tabular}{|c|c|c|c|c|c|c|c|c|c|c|}
\hline \multirow{2}{*}{$\begin{array}{l}\text { Desig- } \\
\text { nation }\end{array}$} & \multicolumn{10}{|c|}{ Branch parameters array } \\
\hline & Begin & End & Code & KPR & $\mathrm{A}$ & B & $\mathrm{C}$ & $\mathrm{D}$ & $\mathrm{E}$ & $\mathrm{U},[\mathrm{kV}]$ \\
\hline EPSS & 0 & 1 & 3 & 1 & $\begin{array}{c}X_{C}=5.54 \\
{[\mathrm{Ohm}]}\end{array}$ & $R_{C}=1.5$ & 0.1 & 0.0 & $115[\mathrm{kV}]$ & 115 \\
\hline$O H P L$ & 1 & 3 & 3 & 1 & $\begin{array}{c}X_{0}=0.413 \\
{[\mathrm{Ohm} / \mathrm{km}]}\end{array}$ & $\begin{array}{c}R_{0}=0.159 \\
{[\mathrm{Ohm} / \mathrm{km}]}\end{array}$ & $\begin{array}{c}l_{13}=59 \\
{[\mathrm{~km}]}\end{array}$ & 0.0 & 0.0 & 115 \\
\hline$T$ & 5 & 7 & 4 & 1 & $\begin{array}{c}U_{S C}=10.5 \\
{[\%]}\end{array}$ & $\begin{array}{c}\Delta P_{S C}=0.01 \\
2[\mathrm{MW}]\end{array}$ & $\begin{array}{l}S_{I}=25 \\
{[\mathrm{MW}]}\end{array}$ & 1.0 & $115[\mathrm{kV}]$ & 10.5 \\
\hline$B S B$ & 6 & 7 & 3 & 0 & $\begin{array}{c}X=0 \\
{[\mathrm{Ohm}]}\end{array}$ & $\begin{array}{c}R=0.001 \\
{[\mathrm{Ohm}]}\end{array}$ & 1.0 & 0.0 & 0.0 & 10.5 \\
\hline$P C L$ & 7 & 9 & 3 & 1 & $\begin{array}{l}X_{0}=0.0263 \\
{[\mathrm{Ohm} / \mathrm{km}]}\end{array}$ & $\begin{array}{l}R_{0}=0.0068 \\
{[\mathrm{Ohm} / \mathrm{km}]}\end{array}$ & $\begin{array}{c}l_{13}=1.5 \\
{[\mathrm{~km}]}\end{array}$ & 0.0 & 0.0 & 10.5 \\
\hline$G L N$ & 0 & 14 & 1 & 1 & $\begin{array}{c}\mathrm{X}^{*}=0.35 \\
{[7]}\end{array}$ & $\mathrm{R}^{*}=0.0[7]$ & $\begin{array}{c}0.8 \\
\text { [MVA] }\end{array}$ & 0.0 & $\begin{array}{c}E^{*}=0.85 \\
{[7]}\end{array}$ & 0.4 \\
\hline
\end{tabular}

\section{Methods of solution and algorithm}

The algorithm of operative calculations of the SC modes is based on method of node voltages and superposition method [4], for which realization the electromotive force (EMF) of the power supplies: infinite power supply, synchronous generators $(S G), S M \mathrm{~s}$, synchronous adaptive compensators $(S A C)$, $I M \mathrm{~s}$ and generalized load nodes $(G L N)$ in specific time instant of the transient should be determined. Then the short current and currents in branches and voltages in given nodes of selected district should be calculated.

The algorithm can be divided into following steps [2]:

1. In first step impedances of all switched-on components of the circuit are calculated, which are reduced to basic conditions in relative or absolute units (it is determined by $K 1$ factor). The impedances are calculated by means of subprogram $Z R X$ (the data form for the subprogram is shown in table 1).

2. At initial time instant of the transient the power supplies are replaced by subtransient impedances and EMFs. EMF of $i$-th PS for $j$-th time instant is computed in terms of Eq. 1 [5]:

$$
E_{*_{i j}}=\sqrt{\left(U_{*_{i(j-1)}}+m_{i} \cdot k_{3 i} \cdot \sin \varphi_{i(j-1)} \cdot X_{*_{i j}}\right)^{2}+\left(k_{3 i} \cdot X_{*_{i j}} \cdot \cos \varphi_{i(j-1)}\right)^{2}},
$$

where $U_{*_{i}(j-1)}$ is voltage across terminals of $i$-th PS at beginning of $j$-th calculative interval. If it cannot be calculated or it is not given, then $U_{*_{i}(j-1)}=1$ in per unit; $m_{i}$ is indicator of $i$-th PS $\left(m_{i}=1\right.$ for SM, $m_{i}=-1$ for IM); $k_{3 i}$ is duty factor of $i$-th PS, if it is not given, then $k_{3 i}=1$.

For subtransient mode $(j=1) \quad X_{i 1}=X_{i}^{\prime \prime}$. If $\sin \varphi_{i(j-1)}$ and $\cos \varphi_{i(j-1)}$ are not given, then $\sin \varphi_{i(j-1)}=\sin \varphi_{R A T i}, \cos \varphi_{i(j-1)}=\cos \varphi_{R A T i}$. For system of infinite power $E_{* \tilde{N}}=1$ for any time instant. If the calculation is carried out in absolute units, then all EMFs are reduced to basic value (in subprogram $Z R X)$ :

$E_{i j}=U_{\text {base }} \cdot E_{*_{i j}}$. Pre-calculated values of EMF are input into branch parameter array.

3. In subprogram $C B M R$ the branches are sorted and the nodes are renumbered is such a way that any branch has a common node with at least one previous branch. And as first the branches with EMF 


\section{MATEC Web of Conferences}

are selected. In the simulation model of the district only the switched-in components $(K P R=1)$ are considered.

4. For initial instant (subtransient mode) of SC $(j=1)$ the reference currents in circuits nodes are determined in terms of:

$$
\dot{I}_{\text {onj }}=\dot{E}_{n j} / \dot{Z}_{i n j},
$$

where $n$ is number of the circuit node, which corresponds to end of a branch with EMF; $\dot{Z}_{\hat{1} n j}$ is impedance of a branch with EMF, i.e. of the branch, starting from «neutral». For all other nodes $\dot{I}_{\text {ónj }}=0$.

5. A matrix of node SC impedances $\left(Z_{s c j}\right)$ is generated by means of subprogram CZMATR, in which the branch expanding method is used [10], and as basic node the node with number «zero» (or «zero node») is accepted.

The method essence is that the initial matrix of generalized parameters of the grid $Z_{s c j}=Z$ is resulted from expanding the grid and eliminating loops after their appearance.

As initial branch the branch that touches the basic node $n(0)$ is selected. And the matrix of node impedances $Z^{(I)}$ for sub-grid, which contains only one branch, has dimension $(1 \times 1)$ and its component is equal to impedance of this branch. Then to initial branch are connected new branches successively one by one, and so the grid is expanding. When new branch is connected, the sub-grid circuit is changed, as well as the dimension of matrix $Z^{(l)}$ and values of its components.

If the connected branch has new node in the sub-grid, then the node impedances matrix $Z^{(l-1)}$ increments its dimension and requires to form components of new line and column. Matrix $Z^{(l-1)}$ is converted into matrix $Z^{(l)}$.

If the connected branch has new loop in sub-grid, then the node impedances matrix $Z^{(l-1)}$ does not change its dimension and all its components are recalculated according to certain formula. Matrix $Z^{(l-1)}$ is converted into matrix $Z^{(l)}$.

The grid expansion can be carried out by using method of tree selection and addition in electrical grid. When connecting to node $b$ of the sub-grid the tree branch $a b$ with impedance $z_{a b}$, the node impedances matrix $Z^{(l-1)}$ is converted into matrix $Z^{(l)}$ in the following way:

$$
Z^{(l)}=\left[\begin{array}{cc}
Z^{(l-1)} & Z_{a}^{(l-1)} \\
Z_{a t}^{(l-1)} & Z_{a a}^{(l-1)}+z_{a b}
\end{array}\right],
$$

Where $Z_{a}^{(l-1)}$ is a sub-matrix composed from components of column of matrix $Z^{(l-1)} ; Z_{a t}^{(l-1)}$ is transposed matrix of $Z_{a}^{(l-1)} ; Z_{a a}^{(l-1)}$ is diagonal component of matrix $Z^{(l-1)}$.

When connecting to basic node $n(0)$ of the sub-grid the tree branch with impedance $z_{a n}$, the matrix $Z^{(l-1)}$ is converted into matrix $Z^{(l)}$ by simpler method:

$$
Z^{(l)}=\left[\begin{array}{cc}
Z^{(l-1)} & 0 \\
0_{a t} & z_{a n}
\end{array}\right],
$$

where 0 is a sub-matrix composed from zero components; $0_{\text {at }}$ is transposed matrix from 0 .

When connecting the addition branch with impedance $z_{a b}$ to the grid inter nodes $a$ and $b$, all components of matrix $Z^{(l-1)}$ are recalculated into components of matrix $Z^{(l)}$ in terms of:

$$
\dot{Z}_{i j}^{l}=\dot{Z}_{i j}^{(l-1)}-\frac{\left(\dot{Z}_{i a}^{(l-1)}-\dot{Z}_{i b}^{(l-1)}\right) \cdot\left(\dot{Z}_{a j}^{(l-1)}-\dot{Z}_{b j}^{(l-1)}\right)}{\dot{Z}_{a a}^{(l-1)}+\dot{Z}_{b b}^{(l-1)}-\dot{Z}_{a b}^{(l-1)}-\dot{Z}_{b a}^{(l-1)}+\dot{Z}_{a b}},
$$

where $\dot{Z}_{i j}^{(l-1)}, \dot{Z}_{i a}^{(l-1)}, \dot{Z}_{i b}^{(l-1)}, \dot{Z}_{a j}^{(l-1)}, \dot{Z}_{b j}^{(l-1)}, \dot{Z}_{a a}^{(l-1)}, \dot{Z}_{b b}^{(l-1)}, \dot{Z}_{a b}^{(l-1)}, \dot{Z}_{b a}^{(l-1)}$ are components of the known matrix $\dot{Z}^{(l-1)} ; \dot{Z}_{i j}^{(l)}$ is component of sought-for matrix $\dot{Z}^{(l)}$. 
When connecting to the sub-grid inter node $a$ and basic node $n$ of addition branch with impedance $z_{a b}$, all components of $Z^{(l-1)}$ are recalculated into components of matrix $Z^{(l)}$ in terms of simpler formula:

$$
\dot{Z}_{i j}^{(l)}=\dot{Z}_{i j}^{(l-1)}-\frac{\dot{Z}_{i a}^{(l-1)} \cdot \dot{Z}_{a j}^{(l-1)}}{\dot{Z}_{a a}^{(l-1)}+\dot{Z}_{a n}}
$$

After finishing the grid expanding the obtained matrix $Z^{(m)}$ will be equal to sought-for matrix $Z$ of generalized parameters of full grid.

6 . Voltages in «load» mode that precedes the SC mode are calculated using node voltages method:

$$
\dot{U}_{\text {InitCond } j}=\dot{Z}_{\mathrm{SC} j} \cdot \dot{I}_{\mathrm{REF} j} \text {, }
$$

where $\dot{Z}_{\mathrm{SC} j}$ is a matrix obtained in item 5 for $j$-th time instant; $\dot{I}_{\mathrm{REF} j}$ is the reference currents vector, whose components are calculated in item 4.

Each of components of the vector-column $\dot{U}_{\text {InitCond } j}$ is determined by:

$$
\dot{U}_{\text {InitCond } i j}=\sum_{n=1}^{N}\left(\dot{Z}_{i n j} \cdot \dot{I}_{\mathrm{REF} n j}\right) \text {, }
$$

where $\dot{Z}_{i n j}$ are impedances, which are components of $i$-the line of matrix $\dot{Z}_{\mathrm{SC} j} ; N$ is number of nodes in the converted circuit, excluding the basic node (0).

7. For a given point the total SC current is calculated in terms of formula:

$$
\dot{I}_{\Sigma k j}=\dot{U}_{\text {InitCond } k j} / \dot{Z}_{\mathrm{CC} j}
$$

where $k$ is number of point of SC, which coincides with number of matrix line $\dot{Z}_{\mathrm{SC} j} ; \dot{Z}_{\mathrm{CC} j}-$ diagonal component of matrix $\dot{Z}_{\mathrm{SC} j}$.

8. Residual voltages in the circuit nodes in SC mode are determined based on superposition of «load» mode on purely «emergency» (additional) mode:

$$
\dot{U}_{\text {Resknj }}=\dot{U}_{\text {InitCond } n j}-\dot{U}_{\text {Add } k n j}=\dot{U}_{\text {Init Cond } n j}-\dot{I}_{k j} \cdot \dot{Z}_{n k j},
$$

where $\dot{U}_{\text {Resknj }}$ is voltage in $n$-th node, when SC is located in $k$, which is the node in «emergency» (additional) mode; $\dot{Z}_{n k j}$ are impedances from $n$-the column and $k$-the line of matrix $\dot{Z}_{\mathrm{SC} j}$.

9. For each SC point the distribution of SC current into branches of the circuit is fulfilled:

- for branch without EMF:

- for branch with PS:

$$
\dot{I}_{k n i j}=\left(\dot{U}_{\text {Resknj }}-\dot{U}_{\hat{1} k i j}\right) / \dot{Z}_{n i j} ;
$$

$$
\dot{I}_{k i i j}=\left(\dot{E}_{i j}-\dot{U}_{i k i j}\right) / \dot{Z}_{i i j} \text {. }
$$

In the software of operative calculation of SC currents all the formulae are realized. For other time instants of SC: $j=2$ (transient mode), $j=3$ (steady - state mode of SC), items $1 \ldots 9$ are repeated.

The described algorithm is realized as software of operative calculations of the SC modes. The calculations of currents for three-phase SC for power supply circuit of Sublimation plant of JSC Siberian Chemical Plant. A part of the parameter array of the branches is shown in table 1 and the corresponding circuit is shown in Fig. 1.

Each branch of the circuit corresponds to one line of table 1. 


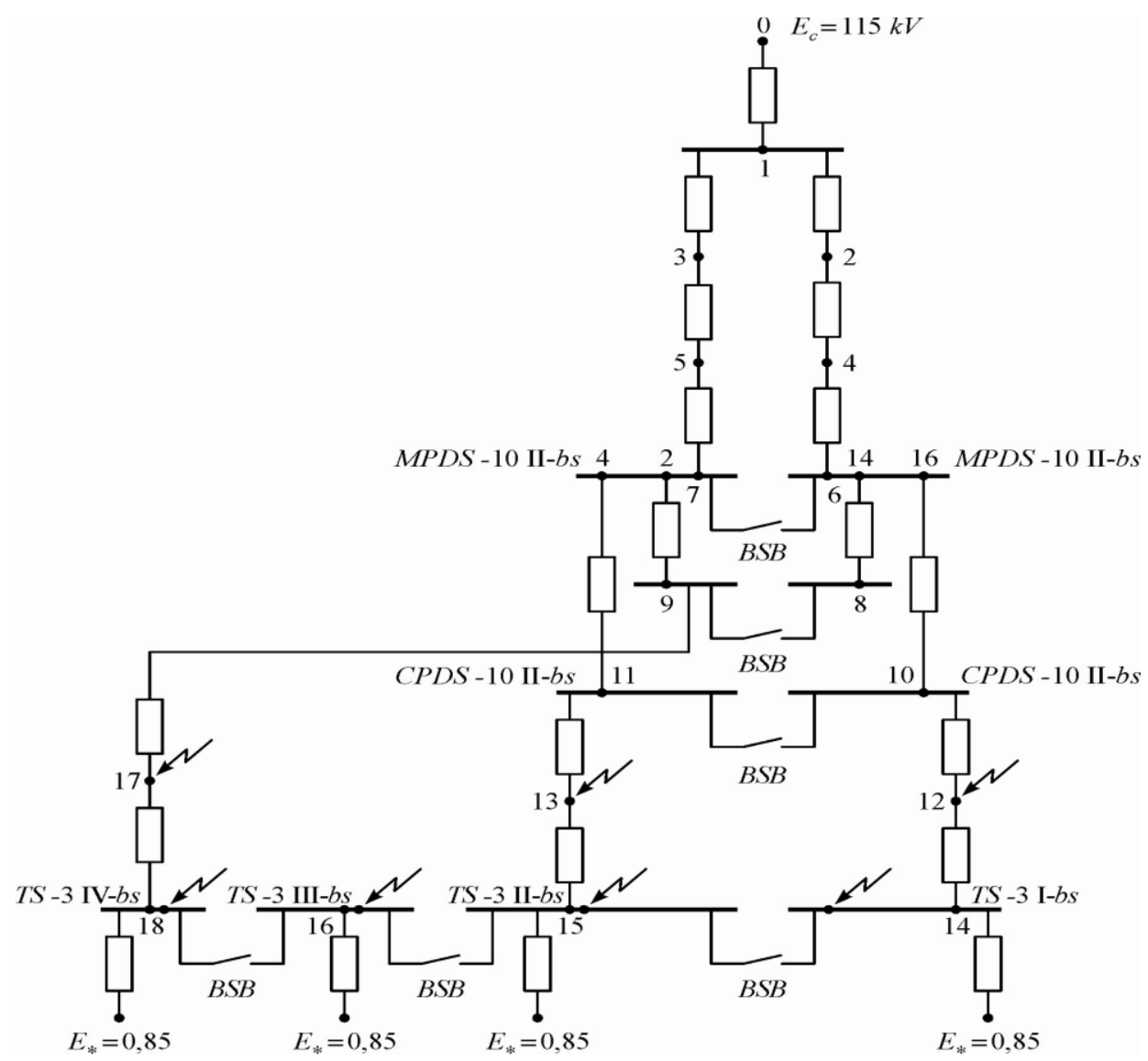

Figure 1. Equivalent circuit of power supply of part of sublimation plant

In Fig. 1 there are following symbols: $M P D S$ is Main Power Distribution Station; $b s$ is bus bar section; $C P D S$ is Central Power Distribution Station; TS is Transformer Station; BSB is Bus Section Breaker.

Table 2. Results of calculation for one point of SC

\begin{tabular}{|c|c|c|c|}
\hline \multicolumn{4}{|c|}{ Point of SC № 12, $\mathrm{I}_{\mathrm{SC}}=9,585 \mathrm{kA}$} \\
\hline (Beginning-end of branch) & Current in branch [kA] & № node & $U_{\text {RES, }[\mathrm{kV}]}$ \\
\hline $0-1$ & 9.477 & 6 & 3.147 \\
\hline $12-14$ & 0.596 & 9 & 9.333 \\
\hline
\end{tabular}

\section{Conclusion}

The software allows to take into account the short circuit inflow from all electrical motors, consider changes in the circuit and calculate residual voltages in nodes and currents in branches of the circuit, which corresponds to requirements of maintenance and tuning of the power supply system [11].

Using the software the rapid analysis of starting and self-starting modes of electric motors can be carried out in order to determine real self-starting (starting) factors, which are part of formula for calculation of operating current of maximum current protection $[8,9]$, and to determine the mounting point of controlled shunt reactors and other voltage regulation means. 
Operative calculations of the SC modes are needed to be carried out for power supply systems with their own power plants [10].

\section{References}

1. Egamnazarov, Georgiy, Proceedings of the 7 th international scientific symposium on Electrical power engineering, 474 (2013)

2. RD 153-34.0-20.527-98. Guidelines for calculation of short circuit currents and selection of electric equipment / Edited by. B.N. Neklepayeva. - M.: Editorial SPO ORGRES, 2003 - 35 p. (in Russian).

3. G. Han, L. Han, L. Wu, Power System Protection and Control, 38 (1), 141 (2010)

4. Kulikov Yu. A. Transients in electrical systems. Textbook (Novosibirsk: NStTU, Moscow: Mir, 2003)

5. Zhukov V.V. Short circuits in nodes of complex load of electrical systems (Moscow: MPEI Publ., 1994).

6. Electrotechnical reference book. Vol. 3. / Ed. by prof. of MPEI V.G. Gerasimov etc. - 8-th Edition, corr. and add (Moscow: MPEI Publ., 2002).

7. Ulyanov S.A. Electromagnetic transients (Moscow, Energiya, 1970).

8. Su Y.R., Yu J., Liu J., Zhou G., Zhan L.P., Adv. Mater. Res. 860-863, 1914 (2014)

9. Shabad M.A. Calculations of relay protection and automatics of distribution networks (Saint Petersburg: PEIPK, 2003).

10. Feng S.-H., Yu Y.-J., Wang L.-W., Shi F., Tang B.-Q., Power System Protection and Control 42 (8), 28 (2014)

11. Gamazin S.I. Transients in industrial electric power supply systems caused by electric motor load (Moscow: MPEI Publ., 1997) 